\title{
Application of mechanical stretch to tune the resonance frequency of hyperelastic membrane-based energy harvesters
}

\author{
Lin Dong ${ }^{1}$, Michael Grissom, M. G. Prasad ${ }^{1}$, and Frank T. Fisher ${ }^{1 *}$
}

\begin{abstract}
Vibration-based energy harvesting has been widely investigated as a means to generate low levels of electrical energy for applications such as wireless sensor networks. However, for optimal performance it is necessary to ensure that resonant frequencies of the device match the target ambient vibration frequencies for maximum energy harvested. Here a novel resonant frequency tuning approach is proposed where the application of membrane stresses generated by different stretch ratios applied to circular hyperelastic membranes is used to tune the vibration response. Specifically, tuning via mechanical stretch is described in terms of effective stiffness theory, where the mechanical stretch of the hyperelastic membrane induces membrane tuning stresses and a corresponding reduction in membrane thickness. A finite element model (FEM) using ANSYS agrees well with an analytical model of the tuned hyperelastic membrane. Lastly, using a mass-loaded circular membrane vibration model, the effective resonant frequency of the energy harvester can be determined as a function of changes in membrane tension due to the applied stretch. Preliminary experiments verify the resonant frequencies predicted from the analytical and FEM models as a function of different levels of mechanical stretch, centrally-loaded added mass, and membrane initial thicknesses. The proposed mechanical stretch tuning approach for hyperelastic membranes provides an alternative tuning strategy to enable energy harvesting from different ambient vibration sources in various environments.
\end{abstract}

Keywords: Energy harvesting, hyperelastic, membrane, vibration, frequency tuning

\section{Introduction}

Energy harvesting is a rapidly growing field which seeks to generate small but useful levels of electrical energy from mechanical vibrations present in most environments. ID Techex [1] summarized the energy harvesting market and discussed potential applications in areas such as healthcare, electronics, and automotive applications, with emerging uses such as a potential power source for micro/nanorobots also envisioned [2]. A particularly compelling application is to replace the batteries of individual sensor nodes comprising wireless sensor networks due to the potential of small size, ease of implementation, and the ability to facilitate the placement of sensors in inaccessible locations, suggesting energy harvesting may have tremendous impact when integrated with ubiquitous wireless microsensors used to provide continuous monitoring of machine and structural health $[3,4]$. A comparative analysis suggests that mechanical vibration shows potential as a high power density and long lifetime energy source among a variety of potential ambient energy harvesting sources [5], and thus a vibration-based energy source is the focus of the current work.

\subsection{Frequency tuning for vibration-based energy harvesting}

A comprehensive discussion of vibration based energy harvesting for wireless, self-powered microsystems is available in the literature [6]. Generally, it is desired to ensure that the resonant frequencies of an energy harvesting device match the ambient vibration frequencies to maximize the energy harvested, and a review of strategies for tuning the frequency range of vibration based energy harvesting has been described elsewhere [7]. For example, Challa et al. [8] presented a resonance 
frequency tuning approach using a magnetic force applied perpendicular to a cantilever beam, achieving $\pm 20 \%$ of the unturned frequency based on the mode (attractive, repulsive) of the magnetic force and the separation distance between the magnets. Zhu's research group [9] designed a horizontal tunable electromagnetic vibration-based micro-generator via induced variable axial magnetic forces. More recently, a two-dimensional resonant frequency magnetic tuning approach was developed which further generalizes these approaches [10]. The tuning model is based on effective stiffness theory, where the effective resonant frequency of the system is related to two variable stiffness terms which the twodimensional placement of the magnets adds to the system. A two-dimensional tuning method may be useful, in particular, for applications where space constraints impact the available design space of the energy harvester. In addition, approaches developed at small length scales to tune the effective stiffness of NEMS and MEMS devices operating at very high frequencies [11-13] may be adapted for energy harvesting applications targeting appropriate frequencies. From a systems-level perspective, one must obviously account for any energy required to tune the harvesting device when evaluating the efficiency and performance of a tunable energy harvesting system [14-16].

In addition to the cantilever-based energy harvesting geometries typically described above, a membrane-based energy harvester could also be implemented. For example, Rezaeisaray et al. [17] designed and analyzed an SU-8 membrane-based energy harvester which uses a nonlinear stiffness effect based on a Duffing oscillator to obtain a frequency bandwidth of $146 \mathrm{~Hz}$. Mo et al. [18] developed a theoretical model for a piezoelectric circular membrane subjected to pressure fluctuations, concluding that the optimization of energy harvesting performance is highly dependent on the ratio between the thickness and radius of the membrane. A piezoelectric circular membrane array connected in parallel was shown by Wang et al. [19] to increase the device performance compared to the power generated from a single membrane. In addition, Palosaari [20] conducted an experimental study that suggests that pressure fluctuations could be a potential method for tuning the resonant frequency of membrane-based energy harvesters. (Note that the term pre-stress used in [20] refers to the membrane out-of-plane stress, which differs from its use to denote the in-plane membrane stress as used below.) Recently, membrane geometry-based energy harvesters have become increasingly attractive as a means to leverage the properties of emerging soft materials, which could be utilized to target lower frequency vibration sources. In particular, hyperelastic polymers with large strain capability are investigated here.

\subsection{Hyperelastic and electroactive membranes}

Piezoelectric materials (including single crystals, ceramics, and polymers) are commonly used materials for vibration-based energy harvesting due to their higher efficiencies and specific power output. In recent years, in addition to those widely used piezoelectric materials, electroactive polymers, which can deform in response to the application of an electrical stimulus, have been explored for potential mechanical energy harvesting applications [21]. Among electroactive polymers, dielectric elastomers (DEAs) have drawn great attention due to their outstanding overall performance [22], including large elongation, high speed of response, and high energy density. Among commercial products [23-26], 3M VHB 4910 acrylic tapes show impressive performance due to their high dielectric constant $\left(\varepsilon_{\mathrm{r}}=4.7\right)$ and the potential for high actuation strain up to 6 times the axial stretch in both planar directions [26] and will be the focus of the work presented here.

Because relatively high strains (well over 100\%) can be produced through mechanical stretch of dielectric elastomers, DEAs typically demonstrate hyperelastic material behavior, where the stress-strain relationship derives from a strain energy function. The mechanical behavior of hyperelastic materials can be characterized by different functional forms of the strain energy, the most common of which are those first described by Yeoh [27], Ogden [28] and Mooney-Rivlin [29]. Previous work found that when using the Ogden and Mooney-Rivlin material models the membrane thickness decreases in an unstable manner, with the resulting membrane stress overestimating the material response [30]. Thus the Yeoh form of the strain energy function is used in this work, with the necessary parameters for the Yeoh hyperelastic material model for 3M VHB 4910 listed in Table 1 [30]. 


\section{Mechanical stretch tuning mechanism}

In this section a model of resonance frequency tuning via mechanical stretch of a hyperelastic membrane-based energy harvester is described. Based on effective stiffness theory, the effects of variable membrane tension on the overall device stiffness are used to tune the effective resonant frequency of the system. Section 2.1 describes the general tuning model for energy harvesting membranes via the application of applied tension. Section 2.2 then presents the relationship between the applied stretch ratio and the membrane tension of a circular hyperelastic membrane. The vibration of a central mass-loaded circular membrane as a function of membrane tuning tension is then presented in Section 2.3, with a theoretical calculation of membrane stretching stiffness derived which can be used in the effective stiffness model to tune the primary resonant frequency of a stretched hyperelastic membrane.

\subsection{Hyperelastic membrane tuning via mechanical stretch}

A theoretical lumped parameter tuning model for stretching of a membrane-based energy harvester is shown in Fig. 1. When the membrane is subjected to mechanical stretch, the membrane radius expands from $r_{0}$ to $r_{1}$, and the thickness contracts from the original thickness $t_{0}$ to the new thickness $t_{1}$. Following the common approach of modeling a vibration-based energy harvesting device as a spring-mass-damper system [31], the tuning model for a stretched membrane-based vibration energy harvester is schematically shown in Fig. 1b. The model consists of a spring of variable stretching stiffness (which can be changed to tune the resonant frequency), a mass, and dampers denoted as mechanical dashpot $b_{m}$ and electrical dashpot $b_{e}$ which represent the mechanical losses and the mechanical energy transformed into electrical energy, respectively. Here the stretching stiffness $k_{\text {stretch }}$ is related to the external applied stretching forces and can be determined as a function of the stretch ratio of the hyperelastic membrane as discussed in Section 2.3 below.

Based on the effective stiffness tuning model, the effective frequency $f_{\text {stretch }}$ of the energy harvester tuned via mechanical stretch of the membrane can be expressed as shown in Eq. (1), where $k_{\text {stretch }}$ is the membrane stretching stiffness and $m_{e f f}$ is the effective mass of the system.

$$
f_{\text {stretch }}=\frac{1}{2 \pi} \sqrt{\frac{k_{\text {stretch }}}{m_{\text {eff }}}}
$$

While in practice it may be difficult to change the added mass during device operation, an alternative approach highlighted in Eq. (1) is to adjust the membrane stretching stiffness in order to target the environmental source frequencies for the maximum power output. (Indeed, the goal of this work is to develop the expression for $k_{\text {stretch }}$ for a hyperelastic membrane subject to mechanical stretch.) Based on the generic lumped parameter model as shown in Fig. 1b, the power output of an energy harvesting device given a sinusoidal ambient excitation vibration $y(t)=Y \sin \omega_{s} t$, (where $Y$ and $\omega_{s}$ are the source vibration amplitude and frequency, respectively) can be written as [31]

$$
P=\frac{m_{\text {eff }} \zeta_{t} Y^{2}\left(\frac{\omega_{s}}{\omega_{\text {struc }}}\right)^{3} \omega_{s}{ }^{3}}{\left[1-\left(\frac{\omega_{s}}{\omega_{\text {struc }}}\right)^{2}\right]^{2}+\left[2 \zeta_{t} \frac{\omega_{s}}{\omega_{\text {struc }}}\right]^{2}}
$$

where total damping ratio $\zeta_{t}\left(\zeta_{t}=\zeta_{m}+\zeta_{e}\right)$ is the sum of mechanical $\zeta_{m}$ and electrical $\zeta_{e}$ damping ratios, respectively, and $\omega_{\text {struc }}$ is the undamped natural frequency of the vibrating structure. Note that the relationship between the damping coefficients of $b_{m}$ and $b_{e}$ as denoted in Fig. $1 \mathrm{~b}$ and the unitless damping ratios of $\zeta_{m}$ and $\zeta_{e}$ used in Eq. (2) are $b_{m}=2 m_{e f f} \zeta_{m} \omega_{s t r u c}$ and $b_{e}=2 m_{e f f} \zeta_{e} \omega_{s t r u c}$. In earlier energy harvesting studies, damping ratios on the order of 0.01 to 0.03 have been reported for 
piezoelectric and electromagnetic devices, respectively [32] while an open circuit Q-factor of 170-195 was reported for a horizontal tunable electromagnetic device [9]. When the energy harvesting device is in resonance such that $\omega_{s}=\omega_{\text {struc }}$, the power output at resonance $P_{\text {res }}$ can be simplified as

$$
P_{r e s}=\frac{m_{e f f} Y^{2} \omega_{s}^{3}}{4 \zeta_{t}}
$$

According to Eq. (3), at resonance the power output is dependent on the effective mass, the amplitude and frequency of the source vibration, and the damping characteristics of the system.

\subsection{Hyperelastic membrane tuning stresses}

When a hyperelastic membrane is subjected to mechanical stretch, the membrane radial stresses are governed by the applied stretch ratio as a function of the hyperelastic constitutive behavior of the material. As discussed previously, the high performance electroactive polymer 3M VHB 4910 acrylic [33] is studied here, with the Yeoh model materials parameters listed in Table 1. Note that the following derivations are simplified by assuming the material is incompressible and isotropic, and the time dependence of the mechanical response is neglected.

The mechanical behavior of hyperelastic materials is characterized by the strain energy function. Based on the Yeoh model [27], the strain energy $W$ depends on the first invariant Cauchy-Green deformation tensor $I_{1}$ such that

$$
W=C_{10}\left(I_{1}-3\right)+C_{20}\left(I_{1}-3\right)^{2}+C_{30}\left(I_{1}-3\right)^{3}
$$

where $C_{10}, C_{20}$ and $C_{30}$ are material parameters and $I_{1}$ can be calculated from the eigenvalues of the deformation gradient tensor for the circular membrane as $I_{1}=\lambda_{r}{ }^{2}+\lambda_{c}{ }^{2}+\lambda_{z}{ }^{2}$, where $\lambda_{r}$ is the stretch ratio in the radial direction while $\lambda_{c}$ and $\lambda_{z}$ are the stretch ratios in circumferential and thickness directions, respectively. Assuming that the material is incompressible and isotropic requires that $\lambda_{r} \cdot \lambda_{c}$. $\lambda_{z}=1$, while for a circular membrane $\lambda_{c}=c_{1} / c_{0}=r_{1} / r_{0}=\lambda_{r}$, where $c_{0}$ and $c_{1}$ are the unstretched and stretched circumference of the membrane, respectively. Thus the first invariant Cauchy-Green deformation tensor $I_{1}$ can be written as $I_{1}=2 \lambda_{r}{ }^{2}+1 / \lambda_{r}{ }^{4}$.

In addition, for an incompressible material, the Cauchy stresses $s_{i}$ are defined as the force per unit area of the deformed configuration. They can be determined based on the derivative of the strain energy potential with respect to the stretch ratios $\lambda_{i}$ and the hydrostatic pressure $p$ which enforces the incompressibility constraint [28].

$$
s_{i}=\lambda_{i} \cdot \frac{\partial W}{\partial \lambda_{i}}-p
$$

where subscript $i$ refers to the different directions for simplification in the following equations. Thus the Cauchy stress in the radial direction is $s_{r}=\lambda_{r} \frac{\partial W}{\partial \lambda_{r}}-p$ and the out-of-plane Cauchy stress in the thickness direction is zero due to the assumption of plane strain for a thin membrane $\left(s_{z}=\lambda_{z} \frac{\partial W}{\partial \lambda_{z}}-p=0\right)$. The Cauchy stress in the radial direction for a hyperelastic circular membrane based on the Yeoh model can be written as

$$
s_{r}=2 \cdot\left(\lambda_{r}^{2}-\frac{1}{\lambda_{r}^{4}}\right) \cdot\left[C_{10}+2 \cdot C_{20} \cdot\left(2 \lambda_{r}^{2}+\frac{1}{\lambda_{r}^{4}}-3\right)+3 \cdot C_{30} \cdot\left(2 \lambda_{r}^{2}+\frac{1}{\lambda_{r}^{4}}-3\right)^{2}\right]
$$

The corresponding contracted thickness of the hyperelastic membrane after stretching is determined as 


$$
t_{1}=t_{0} \cdot \lambda_{z}=\frac{t_{0}}{\lambda_{r}^{2}}
$$

The membrane tension $T$ is a function of the membrane radial stress $s_{r}$ and contracted thickness $t_{1}$ such that $T=s_{r} \cdot t_{1}$.

\subsection{Vibration of a central mass-loaded circular membrane and stretching stiffness}

Earlier a model for the fundamental frequency of a mass-loaded circular membrane was developed as a function of geometry and properties of the membrane, the tension applied to the membrane, and the proof mass at the center of the membrane [34]. This model is summarized here for completeness. Note that, by definition, membranes are always stretched in tension. For a perfectly clamped homogeneous circular membrane, the resonant frequency can be written as [35]

$$
f_{i j}=\frac{\alpha_{i j}}{2 \pi a} \sqrt{\frac{T}{\rho t}} \quad \text { for } i, j=0,1,2,3, \ldots
$$

where $a$ is the radius of the membrane and $T, \rho$, and $t$ are the tension, density, and thickness of the membrane, respectively. Here we are most interested in the first primary vibration mode, as this is the fundamental frequency mode which is generally related to the most energy intensive vibrations. For this first fundamental $(0,1)$ mode, $\alpha_{01}=2.405$ [35]. As expected, according to Eq. (8), the fundamental frequency of the membrane increases with increasing membrane tension.

The natural frequencies of the membrane can be lowered in order to target ambient vibration sources by adding an additional mass to the membrane. Here we assume that the added mass $m$ is spread over a contact radius $b$ at the center of the membrane with a radius of $a$. Here we also assume that the membrane tension $T$ is much larger than the force applied on the membrane by the added mass $m$, such that the effect of the added mass on the tension of the membrane can be neglected. The first fundamental frequency for the membrane with central mass-loaded can then be written as [35]

$$
f=\frac{1}{2 \pi} \sqrt{\frac{2 \pi T}{m \ln \left(\frac{a}{b}\right)}}
$$

where the mass of the membrane is assumed small compared to the added mass $m$ and hence neglected. Note that in the derivation of Eq. (9) $b$ must not be significantly smaller than $a$, as this concentrated "point load" would result in significant stretch in the center of the membrane [35]. The impact of the radius ratio on the vibration response of a centrally-loaded mass circular has been discussed in detail in the literature [34]. According to Eq. (8) and Eq. (9), the frequencies of the unloaded and centrally-loaded membrane are proportional to the square root of the tension. Therefore, tuning the membrane tension could significantly alter the resonant frequency of the device, which would be useful in the development of small footprint energy harvesting devices which are able to target lower environmental source frequencies. An analogous discussion of the resonant frequency of a square membrane with/without added mass for energy harvesting application is presented in [34].

According to Eq. (1), the stretching stiffness of the membrane contributes to the resonant frequency of the membrane-based energy harvester. On the other hand, the vibration response of the membrane with central loaded mass is determined by Eq. (9). Comparing those two equations, it is straightforward to determine the effective mass $m_{\text {eff }}$ and stretching stiffness $k_{\text {stretch }}$ of the central loaded membrane-based energy harvesting system as 


$$
\begin{aligned}
& m_{\text {eff }}=m \cdot \ln \left(\frac{a}{b}\right) \\
& k_{\text {stretch }}=2 \pi \cdot T
\end{aligned}
$$

Given that the membrane tension $T$ can be written as $T=s_{r} \cdot t_{1}$, the stretching stiffness of a circular hyperelastic membrane as a function of stretch ratio $\lambda$ can be derived by substituting Equations (6) and (7) into Eq. (11) such that

$$
k_{\text {stretch }}=4 \pi \cdot t_{0} \cdot\left(1-\frac{1}{\lambda_{r}^{6}}\right) \cdot\left[C_{10}+2 \cdot C_{20} \cdot\left(2 \lambda_{r}^{2}+\frac{1}{\lambda_{r}^{4}}-3\right)+3 \cdot C_{30} \cdot\left(2 \lambda_{r}^{2}+\frac{1}{\lambda_{r}^{4}}-3\right)^{2}\right]
$$

\section{Finite element modeling approach}

To verify the theoretical calculations discussed in Section 2, two separate FEM simulations using ANSYS were performed for the circular hyperelastic membrane as described in this section. In the first analysis using ANSYS, the membrane tuning stresses was first determined as a function of the applied mechanical stretch to apply a desired tension to the membrane. This result then forms the basis for the second FEM step, where a modal analysis simulation is used to determine the resonant frequencies of the central mass-loaded membrane.

According to Eq. (6), it is clear that the Cauchy stress in the radial direction $s_{r}$ is only related to the stretch ratio $\lambda$ and the material properties $\left(C_{10}, C_{20}\right.$ and $\left.C_{30}\right)$. Verifications of membrane tuning stresses are performed by using ANSYS as shown in Fig. 2. The hyperelastic membrane (3M VHB 4910) is modeled using the element SOLID 226 with KEYOPT (11) set to 1 to characterize a fully incompressible elastomer. As shown in Fig. 2, the displacement of the nodes at the model boundary are imposed from the initial radius $r_{0}$ to a stretched radius $r_{1}=\lambda_{r} \cdot r_{0}$, with cyclic symmetry boundary conditions in cylindrical coordinates with an opening angle of thirty degrees are used in the FEM analysis. The material properties for the Yeoh model are used as shown in Table 1, where a membrane initial radius $r_{0}$ of $19.05 \mathrm{~mm}$ is used to correspond to the experimental data (will discuss in Section 4). Therefore, the membrane radial stresses for a given value of stretch $\lambda$ are determined by averaging the radial stresses at the exterior nodes where $r=r_{1}$. In addition, based on the resulting displacement for the nodes of the membrane, the contracted thickness of the membrane can be determined as the average value of the difference in displacements between the membrane top and bottom layers.

Once the analysis for the membrane radial stress has been completed, this result is then used to determine the resonant frequency of the stretched membrane (with a central proof mass added at this stage) for comparison with the theoretical calculations of membrane tuned frequency as shown in Fig. 3. The membrane with central loaded mass is modeled using ANSYS Shell 41 elements asymmetrically with an opening angle of thirty degrees and symmetric boundary conditions as shown in Fig. 4. A radius ratio between the membrane and the central load of $a / b=9$ was used for the FEM analysis to enable comparison with the experimental study described in the next section. In the analysis for the centrallyloaded membrane, a static analysis is first performed to apply the desired tension calculated from the initial FEM analysis as a function of the stretch ratio. Specifically, this membrane stress is induced by the method of uniform cooling, where the temperature difference $\Delta T_{\text {temp }}$ is calculated from $\Delta T_{\text {temp }}=$ $T /(E \alpha t)$ [36], where $T$ is the tension of the membrane and $E$ and $\alpha$ are the Young's modulus and thermal expansion coefficient of the membrane, respectively. Details of the parameters used in the ANSYS study are given in Table 1. Then the next step of the process is that the modal analysis of the clamped membrane with the added central mass is performed. In this second ANSYS model, the added mass attached to the central section of the membrane is modeled as two sets of overlapping elements. The first 
set of elements representing the membrane (lower layer) has its nodal plane located on the 'top' face, whereas the set of elements corresponding to the added mass (top layer) has its nodal plane located on the 'bottom' face. The combination of overlapping elements thus defines the attachment of this layered membrane with its nodal plane at the interface between the layers.

\section{Experimental methods}

A preliminary experimental study measuring the resonant frequency of mass-loaded circular membranes subjected to different stretch ratios is conducted to confirm the results from the analytical and computational models described above. As discussed before, the membrane material used is $3 \mathrm{M}$ VHB 4910 acrylic with the material properties are given in Table 1. Experimental data was obtained for two commercially available initial film thicknesses of $500 \mu \mathrm{m}$ and $1000 \mu \mathrm{m}$, respectively, which were stretched to final thicknesses of $20 \mu \mathrm{m}$ and $111 \mu \mathrm{m}$, respectively.

Tests to determine the effective resonant frequencies of the membranes were performed by mounting a prototype on a small shaker as shown in Fig. 5. The prototype consisted of a membrane, a central magnet/mass, and a coil fixed to the frame which is mounted on a shaker (Vibration Test Systems VG100A-6) to provide a given vibration of known amplitude and frequency. The mass at the center of the membrane was varied by adding multiples of 7 gram ferrous masses (for a total added mass of 14, 28 and 42 grams) to the central magnet. The resonance for the mass-membrane system was evaluated by measuring the frequency response function of the voltage induced in the coil relative to the input force measured by a dynamic load cell (PCB 208A02) mounted between the shaker and the prototype using a high impedance measurement device (NI-9215). The peak of the frequency response function is recorded as an estimate of the resonance of the system.

\section{Results and discussion}

\subsection{Stretching stiffness for the membrane}

The results of the membrane radial stress $s_{r}$ obtained both theoretically and from FEM as a function of stretch ratio are shown in Fig. 6a. Note that because the stretch ratios are always larger than one $\left(\lambda_{r}=r_{1} / r_{0}>1\right)$, the ANSYS simulations are performed starting $\lambda_{r}=1.1$. From Fig. 6a, the theoretical calculations of membrane radial stresses after stretching based on Eq. (6) agree well with the FEM simulations in ANSYS. In addition, it can be also be observed in Fig. 6a that the radial stresses of the hyperelastic membrane grow faster at larger stretch ratios, suggesting that a large stretch ratio may be desired to use in order to widen the frequency tuning range for the membrane-based energy harvesting applications. In addition, the result of the membrane contracted thickness as function of stretch ratio is shown in Fig. 6b, which shows that a membrane with an initial thickness of $1000 \mu \mathrm{m}$ has a membrane thickness of $444.4 \mu \mathrm{m}$ at a stretch ratio of 1.5 and the membrane thickness decreases to $40 \mu \mathrm{m}$ at stretch ratio of 5. Again, the FEM results agree very well with the analytical solution.

Furthermore, the theoretical result of membrane stretching stiffness $k_{\text {stretch }}$ based on Eq. (12) is shown in Fig. 7. A characteristic that can be observed in Fig. 7 is that the stretching stiffness initially increases at small stretch ratios $\left(1<\lambda_{r}<1.5\right)$, while for intermediate stretch ratios between 2 and 3.5 the membrane stretching stiffness exhibits a significant non-linearity and at some points actually decreases as a function of stretch. For larger stretch ratios $\left(\lambda_{r}>3.5\right)$, the membrane stretching stiffness again increases significantly as a function of stretch. The result from Fig. 7 is due to the contributions of both the membrane radial stress and the contracted membrane thickness on the membrane stretching stiffness $k_{\text {stretch }}$. At lower stretch ratios $\left(1<\lambda_{r}<1.5\right)$, the stretching stiffness of the hyperelastic membrane $k_{\text {stretch }}$ increases with increasing stretch ratios. When the stretch ratio is between 2 and 3.5, the decrease in the membrane contracted thickness compensates for the slight increase of the membrane radial stresses, resulting in the nonlinear effective stiffness observed in this range. For larger stretch ratios $\left(\lambda_{r}>3.5\right)$, the membrane radial stresses are dramatically increasing while the membrane thickness gradually decreases, resulting in the observed increasing stretching stiffness of the hyperelastic membrane. The results shown in Fig. 7 suggest that a large stretch ratio $\left(\lambda_{r}>3.5\right)$ could be desired for tuning of the 
hyperelastic membrane-based energy harvesting applications. Thus, in the experimental study described in Section 4, representative values of stretch ratio of 3, 4 and 5 are used to measure the resonant frequency of a mass-loaded circular hyperelastic membrane.

We note that while the theoretical result of membrane stretching stiffness shown in Fig. 7 showed a potential tuning range for stretch ratio of $1<\lambda_{r}<1.5$, this analysis only considers a membrane that is initially stretched prior to adding the centrally loaded mass. The stretching stiffness is only a function of the stretch ratio and membrane material properties based on Eq. (12). However, as will be discussed in the next section, a key assumption in the analytical model is that the impact of the added mass is small compared to the applied tension resulting from the stretch in order to calculate the effective tuned frequency of the membrane with centrally loaded mass from Eq. (9). Thus the larger range of stretch ratios $\left(\lambda_{r}>3.5\right)$ appears most promising for energy harvesting tuning application, resulting in the largest change in stretching stiffness as a function of applied stretch with the behavior monotonic in this region.

\subsection{Membrane tuning frequency with added mass}

Based on the effective stiffness theory described in Section 2, one can theoretically determine the effective resonant frequency of a mass-loaded circular membrane subjected to a mechanical stretch as a function of different added masses, initial thicknesses and stretch ratios using Eq. (1), (10), and (12). These theoretical predictions are compared in Fig. 8 with the results from the finite element analysis and two small sets of experimental data for the hyperelastic membranes listed in Table 2. A radius ratio of $a / b=9$ matching the experimental conditions was used for the analytical predictions. (A discussion of the impact of radius ratio on the effective resonant frequency of the membrane is presented elsewhere [34].) Values for the membrane stretch ratio of 3, 4, and 5 were used in the analytical model.

As shown in Fig. 8, the FEM results agree well with the theoretical model. For example, Fig. 8a shows that a larger centrally-loaded mass can significantly decrease the first natural frequency of the loaded membrane, with experimental results agreeing with the theoretical results very well. On the other hand, if the membranes are subjected to the same stretch ratio, it is clear from Fig. 8b that the lower initial membrane thickness corresponds to a lower resonant frequency.

In addition, the effective tuned resonant frequencies as a function of stretch ratio for the two sets of films with initial thicknesses of $500 \mu \mathrm{m}$ and $1000 \mu \mathrm{m}$ are shown in Fig. 9a and Fig 9b, respectively. As expected based on Eq. (1), the tuned resonant frequency of the membrane has a shape similar to that of the membrane stretching stiffness shown in Fig. 7. Specifically, for the same initial thickness of the membrane, at larger stretch ratios $\left(\lambda_{r}>3.5\right)$, increasing the membrane tension (i.e. increasing the stretch ratio) can effectively increase the first resonant frequency of the loaded membrane as shown in Fig. 9. We note that previous work for hyperelastic films has found that experimental factors such as temperature and the preparation and history of test samples can influence the effective properties of the materials [26, 37]. The results shown in Fig. 9 suggest that mechanical stretch shows promise as a means of providing an appreciable bandwidth for tuning the resonant frequency of membrane-based energy harvesting devices.

Lastly, the experimental frequency results are compared with analytical calculations as a function of the ratio of membrane tension to the force induced by the added mass in Fig. 10. The experimentally obtained frequencies in most cases closely match the analytical model, with the largest differences found for the cases of small ratios of membrane tension to the forces induced by the added mass $(\mathrm{T} / \mathrm{mg})$. This error is attributed to the fact that, for smaller ratios of $T / m g$, the force induced by the added mass is comparable with the membrane tension, such that in those cases the added mass can appreciably impact the tension of the membrane. Due to the assumptions implicit in the development of the analytical model, Eq. (9) does not as accurately capture the results from the experiments in those cases. The results in Fig. 10 suggest that large ratios of $T / \mathrm{mg}$ can be used for the design of tunable hyperelastic membrane-based energy harvesters with a targeted resonant frequency via mechanical stretch.

\section{Conclusions}

Given the increasing interest in the development of wireless sensor networks, technologies which could provide the self-contained sensors with their own renewable power supply are becoming increasing 
attractive. Moreover, recent advances in low-power sensor technology have reduced the power requirements of these sensor nodes, thus it is becoming increasingly feasible to implement wireless sensors self-powered by non-conventional power sources, of which vibration-based energy harvesters are possible solutions. Practical implementation of vibration-based energy harvesters will almost certainly require a way to tune the resonant frequencies to enable the device to harvest energy in various environments. In the current work, a resonant frequency tuning approach for a hyperelastic circular membrane-based energy harvester is developed based on effective stiffness theory. Specifically, the effect of the mechanical stretch on the hyperelastic membrane induces a membrane tuning stress and corresponding reduction in membrane thickness which impacts the effective stiffness and hence the vibrational response of the system. Finite element modeling in ANSYS verified the predictions obtained via the analytical model, with both approaches well describing the data obtained from an experimental study of a centrally-loaded hyperelastic membrane-based energy harvester. We note that a limitation of the proposed tuning approach is that such a technique may be difficult to practically implement while the device is in operation (i.e. adaptive, real-time tuning of the membrane resonant frequency). In addition, note that from a systems perspective, the energy needed to implement the tuning mechanism (in this case, the effective work done by the actuator for stretching) should be included in an overall analysis of the system performance [16]. Nonetheless, these results can be used to guide the design of a tunable membrane-based hyperelastic energy harvester via the application of membrane stresses to enable one to match the resonant frequencies of the harvesting mechanism with the ambient source vibration frequencies present in a given environment.

\section{Acknowledgements}

This work was supported in part by the Defense Threat Reduction Agency [contract \# HDTRA1-11P-0023]. The authors also acknowledge the financial support of the Innovation and Entrepreneurship Doctoral Fellowship program at Stevens.

\section{References}

[1] P. Harrop and R. Das, "Energy Harvesting and Storage for Electronic Devices 2010-2020," IDTechEx, 2010.

[2] J. Ding, V. R. Challa, M. G. Prasad, and F. T. Fisher, "Vibration Energy Harvesting and Its Application for Nano- and Microrobotics," in Selected Topics in Micro/Nano-robotics for Biomedical Applications, Y. Guo, Ed., ed New York, NY: Springer New York, 2013, pp. 59-83.

[3] G. Park, T. Rosing, M. Todd, C. Farrar, and W. Hodgkiss, "Energy harvesting for structural health monitoring sensor networks," Journal of Infrastructure Systems, vol. 14, pp. 64-79, 2008.

[4] A. Bengi and G. Vehbi Cagri, "Wireless sensor networks for structure health monitoring: recent advances and future research directions," Sensor Review, vol. 31, pp. 261-276, 2011.

[5] S. J. Roundy, P. K. Wright, and J. Rabaey, "A study of low level vibrations as a power source for wireless sensor nodes," Computer Communications, vol. 26, pp. 1131-1144, 2003.

[6] S. P. Beeby, M. J. Tudor, and N. M. White, "Energy harvesting vibration sources for microsystems applications," Measurement Science and Technology, vol. 17, pp. R175-R195, 2006.

[7] D. Zhu, M. J. Tudor, and S. P. Beeby, "Strategies for increasing the operating frequency range of vibration energy harvesters: a review," Measurement Science and Technology, vol. 21, pp. 022001, 2010.

[8] V. R. Challa, M. G. Prasad, Y. Shi, and F. T. Fisher, "A vibration energy harvesting device with bidirectional resonance frequency tunability," Smart Materials and Structures, vol. 17, pp. 015035, 2008.

[9] D. Zhu, S. Roberts, M. J. Tudor, and S. P. Beeby, "Design and experimental characterization of a tunable vibration-based electromagnetic micro-generator," Sensors and Actuators A: Physical, vol. 158, pp. 284-293, 2010. 
[10] L. Dong, M. G. Prasad, and F. T. Fisher, "Two-dimensional resonance frequency tuning approach for vibration-based energy harvesting," Smart Materials and Structures, vol. 25, pp. 065019, 2016.

[11] N. Manca, L. Pellegrino, T. Kanki, S. Yamasaki, H. Tanaka, A. S. Siri, et al., "Programmable mechanical resonances in MEMS by localized Joule heating of phase change materials," Advanced Materials, vol. 25, pp. 6430-6435, 2013.

[12] M. V. Vitorino, S. Carpentier, A. Panzarella, M. S. Rodrigues, and L. Costa, "Giant resonance tuning of micro and nanomechanical oscillators," Scientific Reports, vol. 5, pp. 7818, 2015.

[13] M. de Laat, H. Pérez Garza, and M. Ghatkesar, "In situ stiffness adjustment of AFM probes by twoorders of magnitude," Sensors, vol. 16, pp. 523, 2016.

[14] Y. C. Shu and I. C. Lien, "Efficiency of energy conversion for a piezoelectric power harvesting system," Journal of Micromechanics and Microengineering, vol. 16, pp. 2429-2438, 2006.

[15] D. Zhu, S. Roberts, J. Tudor, and S. Beeby, "Closed loop frequency tuning of a vibration-based micro-generator," in PowerMEMS 2008+ microEMS2008, Sendai, Japan, 2008, pp. 229-232.

[16] V. R. Challa, M. G. Prasad, and F. T. Fisher, "Towards an autonomous self-tuning vibration energy harvesting device for wireless sensor network applications," Smart Materials and Structures, vol. 20, pp. 025004, 2011.

[17] M. Rezaeisaray, M. E. Gowini, D. Sameoto, D. Raboud, and W. Moussa, "Wide-bandwidth piezoelectric energy harvester with polymeric structure," Journal of Micromechanics and Microengineering, vol. 25, pp. 015018, 2015.

[18] C. Mo, J. Davidson, and W. W. Clark, "Energy harvesting with piezoelectric circular membrane under pressure loading," Smart Materials and Structures, vol. 23, pp. 045005, 2014.

[19] W. Wang, T. Yang, X. Chen, X. Yao, and Q. Zhou, "Vibration energy harvesting using piezoelectric circular diaphragm array," in Applications of Ferroelectrics (ISAF/PFM), International Symposium on Piezoresponse Force Microscopy and Nanoscale Phenomena in Polar Materials, Vancouver, Canada, 2011, pp. 1-4.

[20] J. Palosaari, M. Leinonen, J. Juuti, J. Hannu, and H. Jantunen, "Piezoelectric circular diaphragm with mechanically induced pre-stress for energy harvesting," Smart Materials and Structures, vol. 23, pp. 085025, 2014.

[21] R. D. Kornbluh, R. Pelrine, H. Prahlad, A. Wong-Foy, B. McCoy, S. Kim, et al., "Stretching the Capabilities of Energy Harvesting: Electroactive Polymers Based on Dielectric Elastomers," in Advances in Energy Harvesting Methods, N. Elvin and A. Erturk, Eds., ed New York, NY: Springer New York, 2013, pp. 399-415.

[22] Y. Bar-Cohen, Electroactive Polymer (EAP) Actuators as Artificial Muscles: Reality, Potential, and Challenges. Washington: SPIE Press, 2004.

[23] G. Gallone, F. Galantini, and F. Carpi, "Perspectives for new dielectric elastomers with improved electromechanical actuation performance: Composites versus blends," Polymer International, vol. 59, pp. 400-406, 2010.

[24] M. Y. Benslimane, H.-E. Kiil, and M. J. Tryson, "Dielectric electro-active polymer push actuators: performance and challenges," Polymer International, vol. 59, pp. 415-421, 2010.

[25] R. D. Kornbluh, R. Pelrine, Q. Pei, R. Heydt, S. Stanford, S. Oh, et al., "Electroelastomers: applications of dielectric elastomer transducers for actuation, generation, and smart structures," in SPIE Industrial and Commercial Applications of Smart Structures Technologies, San Diego, CA, 2002, pp. 254-270.

[26] G. Kofod, "Dielectric Elastomer Actuators," Ph.D. Thesis, Technical University of Denmark, Kongens Lyngby, Denmark, 2001.

[27] O. H. Yeoh, "Characterization of elastic properties of carbon-black-filled rubber vulcanizates," Rubber Chemistry and Technology, vol. 63, pp. 792-805, 1990.

[28] R. W. Ogden, "Large deformation isotropic elasticity: on the correlation of theory and experiment for compressible rubberlike solids," Proceedings of the Royal Society of London A: Mathematical, Physical and Engineering Sciences, vol. 328, pp. 567-583, 1972. 
[29] M. Mooney, "A theory of large elastic deformation," Journal of Applied Physics, vol. 11, pp. 582892, 1940.

[30] M. Wissler and E. Mazza, "Modeling of a pre-strained circular actuator made of dielectric elastomers," Sensors and Actuators A: Physical, vol. 120, pp. 184-192, 2005.

[31] C. B. Williams and R. B. Yates, "Analysis of a micro-electric generator for microsystems," Sensors and Actuators A: Physical, vol. 52, pp. 8-11, 1996.

[32] V. R. Challa, M. G. Prasad, and F. T. Fisher, "A coupled piezoelectric-electromagnetic energy harvesting technique for achieving increased power output through damping matching," Smart Materials and Structures, vol. 18, pp. 095029, 2009.

[33] R. Pelrine, R. Kornbluh, Q. Pei, and J. Joseph, "High-speed electrically actuated elastomers with strain greater than 100\%," Science, vol. 287, pp. 836-839, 2000.

[34] L. Dong, M. Grissom, and F. T. Fisher, "Resonant frequency of mass-loaded membranes for vibration energy harvesting applications," AIMS Energy, vol. 3, pp. 344-359, 2015.

[35] N. H. Fletcher, Acoustic Systems in Biology. New York, NY: Oxford University Press, Inc, 1992.

[36] S. Timoshenko and D. H. Young, Vibration Problems in Engineering, 3rd ed. New York, NY: D. Van Nostrand Co., Inc, 1955.

[37] G. Kofod, "The static actuation of dielectric elastomer actuators: how does pre-stretch improve actuation?," Journal of Physics D: Applied Physics, vol. 41, pp. 215405, 2008. 


\section{Captions}

Fig 1. (a) Schematic of tuning model via mechanical stretch of a membrane-based energy harvester. (b) Lumped parameter model where the stretching stiffness is represented as a variable spring and is a function of the applied membrane stresses.

Fig 2. ANSYS model of a circular hyperelastic membrane subjected to a mechanical stretch.

Fig 3. Flow chart of finite element modeling using ANSYS for the circular hyperelastic membrane.

Fig 4. A schematic model of a circular membrane with a centrally loaded mass in ANSYS.

Fig 5. (a) Schematic of the resonant frequency test for a circular hyperelastic membrane with a centrallyloaded (magnetic) mass. (b) Picture of the prototype mounted to the shaker.

Fig 6. Impact of mechanical stretch on circular hyperelastic membrane radial stress and thickness: (a) radial stress as a function of stretch ratio; (b) thickness as a function of stretch ratio.

Fig 7. Stretching stiffness of a circular hyperelastic membrane as a function of stretch ratio based on Eq. (12). The initial (unstretched) membrane thickness is $1000 \mu \mathrm{m}$.

Fig 8. Comparison of analytical predictions (black lines), experimental data (squares) and FEM results (dashed lines) for the effective first mode natural frequency of a circular mass-loaded hyperelastic membrane subjected to mechanical stretch: (a) frequency as a function of added mass; (b) frequency as a function of membrane initial thickness.

Fig 9. Comparison of analytical predictions (black lines), experimental data (squares) and FEM results (dashed lines) for the effective first mode natural frequency of a circular mass-loaded hyperelastic membrane as a function of stretch ratio subjected to mechanical stretch: (a) the initial (unstretched) membrane thickness is $500 \mu \mathrm{m}$; (b) the initial (unstretched) membrane thickness is $1000 \mu \mathrm{m}$.

Fig 10. Comparison of analytical predictions and experimental data for the effective first mode natural frequency of a circular mass-loaded membrane subject to different stretch ratios.

Table 1. Material parameters of hyperelastic membrane.

Table 2. Experimental conditions for measurement of the tuned frequency of stretched hyperelastic $3 \mathrm{M}$ VHB 4910 membranes. The initial unstretched radius of all membranes is $19.05 \mathrm{~mm}$. 


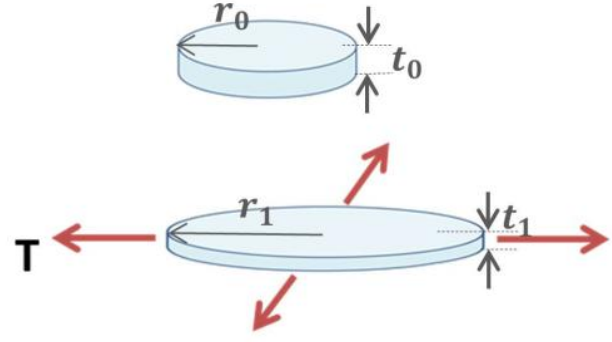

(a)

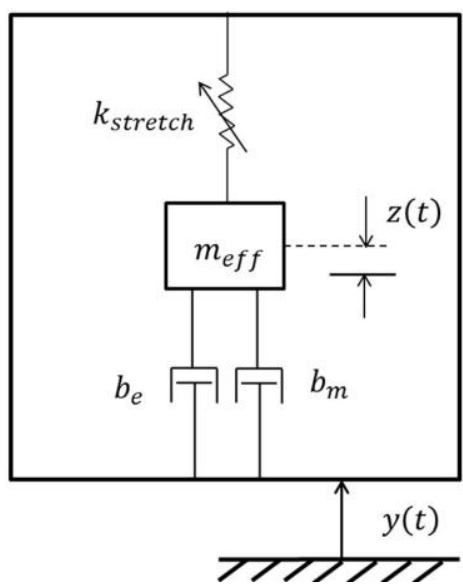

(b)

Fig 1. (a) Schematic of tuning model via mechanical stretch of a membrane-based energy harvester. (b) Lumped parameter model where the stretching stiffness is represented as a variable spring and is a function of the applied membrane stresses. 


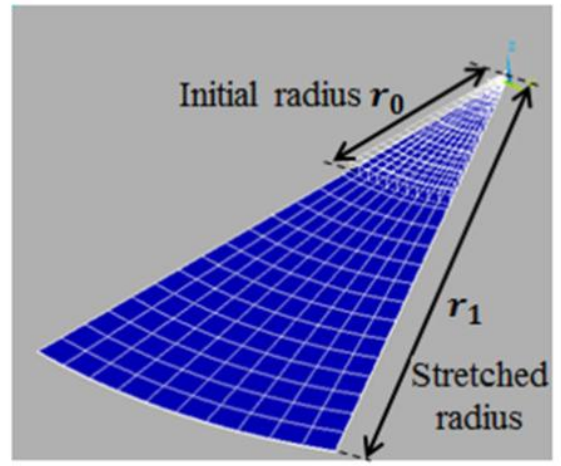

Fig 2. ANSYS model of a circular hyperelastic membrane subjected to a mechanical stretch. 
Simulation 1

Unstretched

Pure Membrane

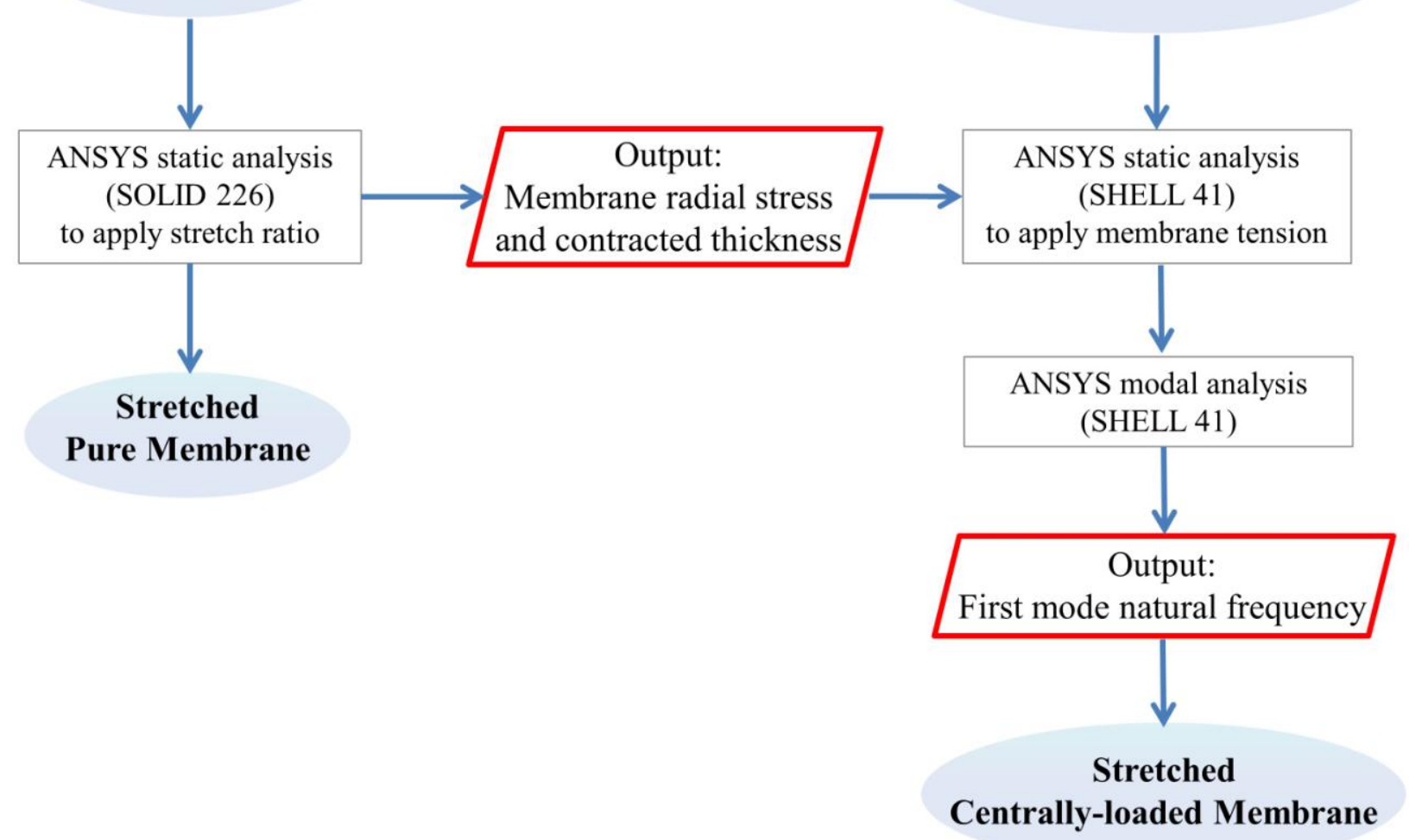

Fig 3. Flow chart of finite element modeling using ANSYS for the circular hyperelastic membrane. 


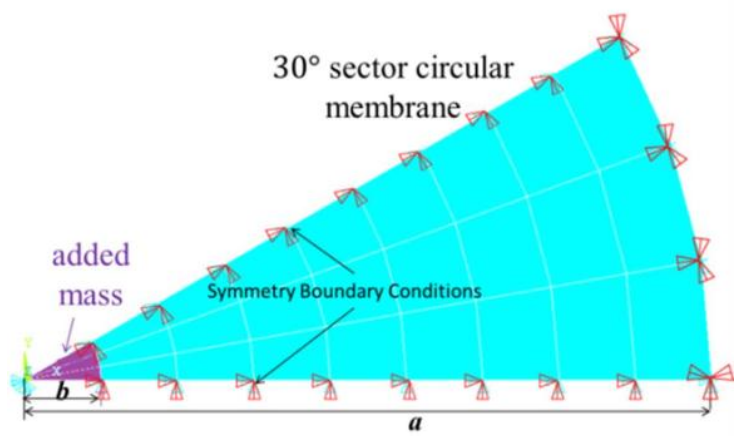

Fig 4. A schematic model of a circular membrane with a centrally loaded mass in ANSYS. 


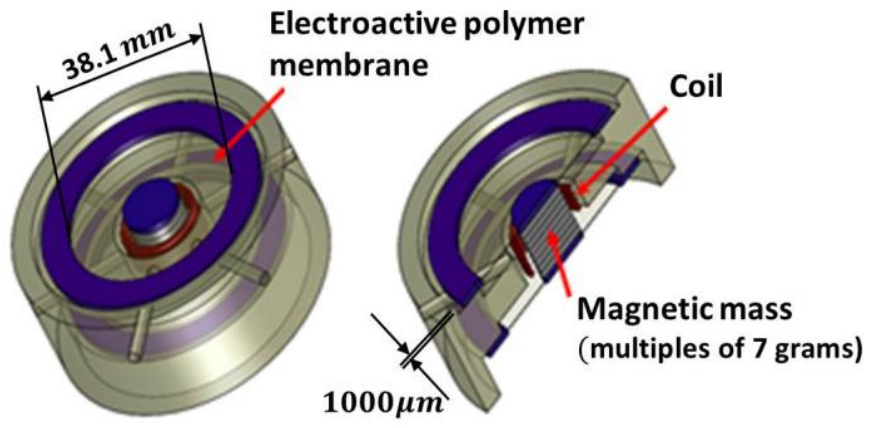

(a)

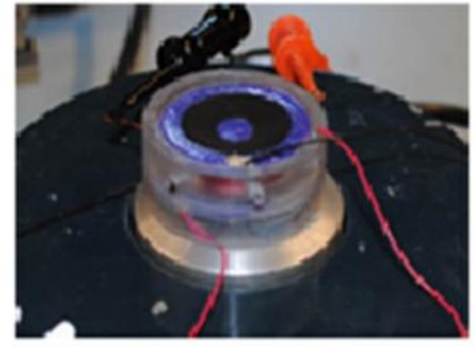

(b)

Fig 5. (a) Schematic of the resonant frequency test for a circular hyperelastic membrane with a centrallyloaded (magnetic) mass. (b) Picture of the prototype mounted to the shaker. 


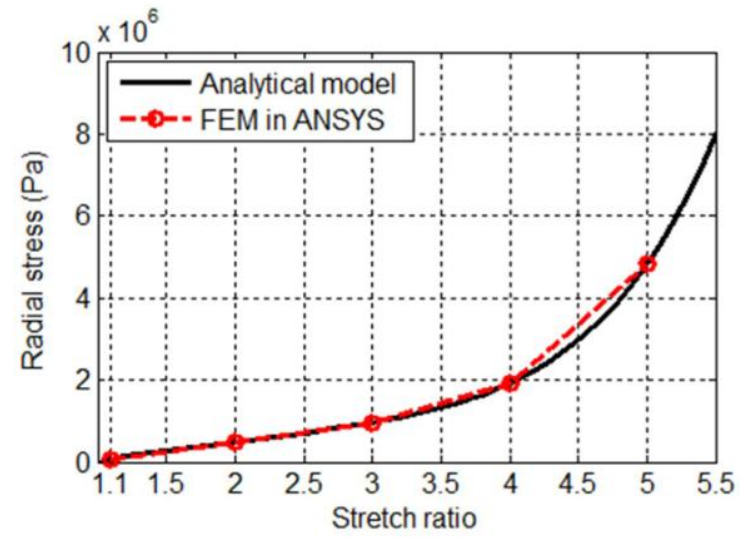

(a)

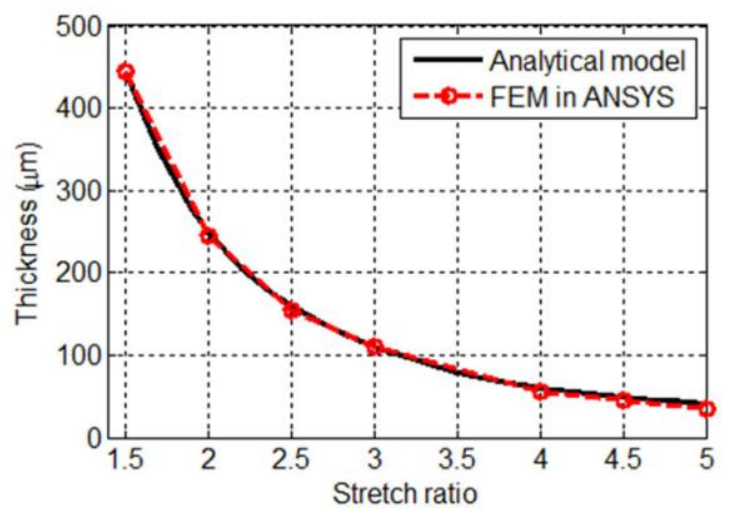

(b)

Fig 6. Impact of mechanical stretch on circular hyperelastic membrane radial stress and thickness: (a) radial stress as a function of stretch ratio; (b) thickness as a function of stretch ratio. 


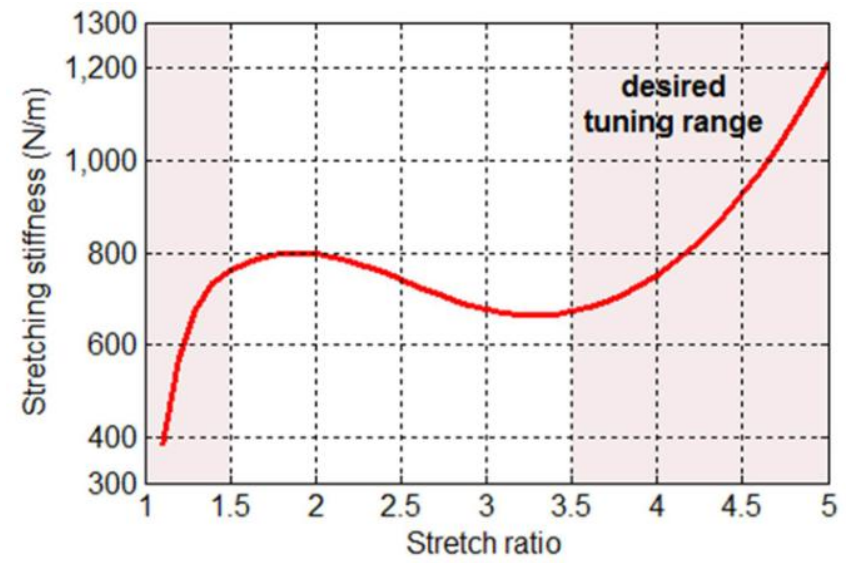

Fig 7. Stretching stiffness of a circular hyperelastic membrane as a function of stretch ratio based on Eq. (12). The initial (unstretched) membrane thickness is $1000 \mu \mathrm{m}$. 


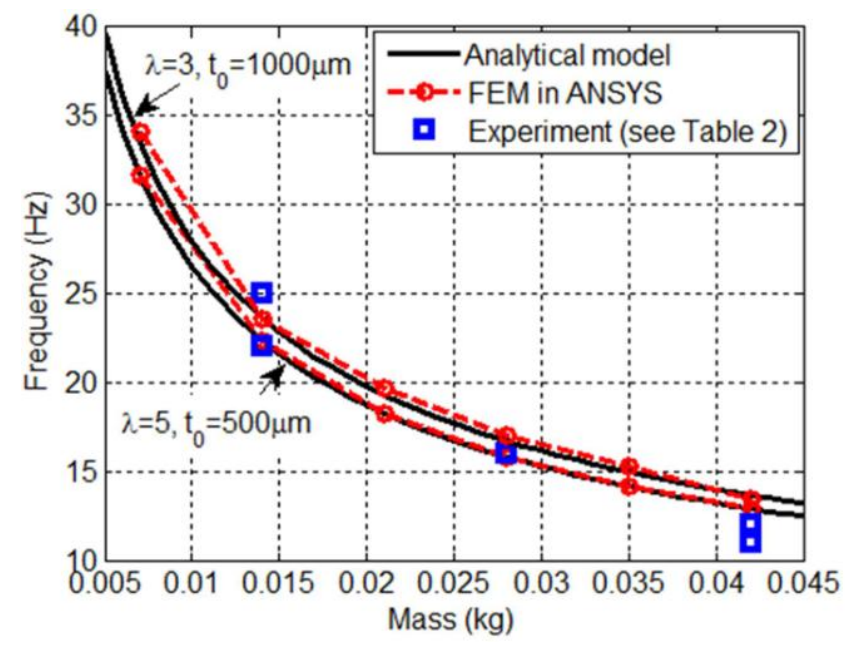

(a)

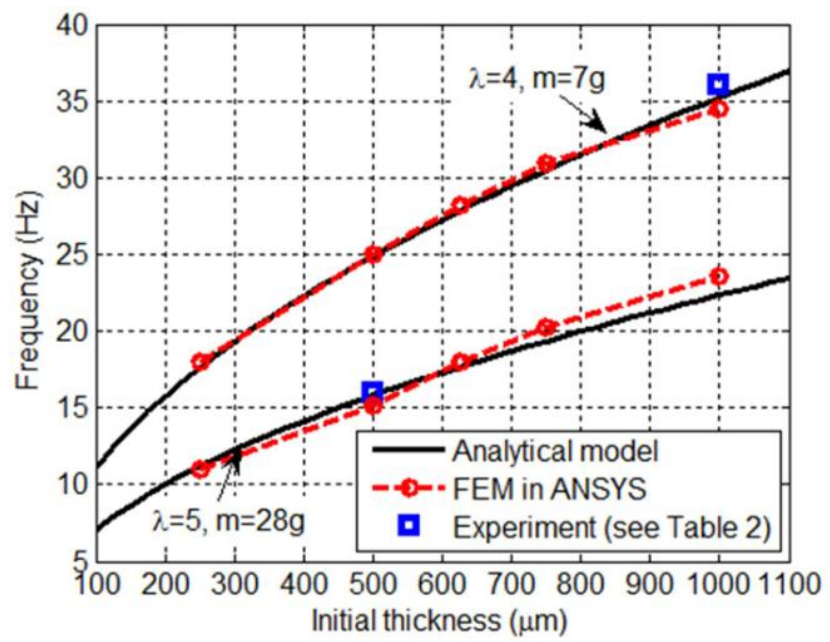

(b)

Fig 8. Comparison of analytical predictions (black lines), experimental data (squares) and FEM results (dashed lines) for the effective first mode natural frequency of a circular mass-loaded hyperelastic membrane subjected to mechanical stretch: (a) frequency as a function of added mass; (b) frequency as a function of membrane initial thickness. 


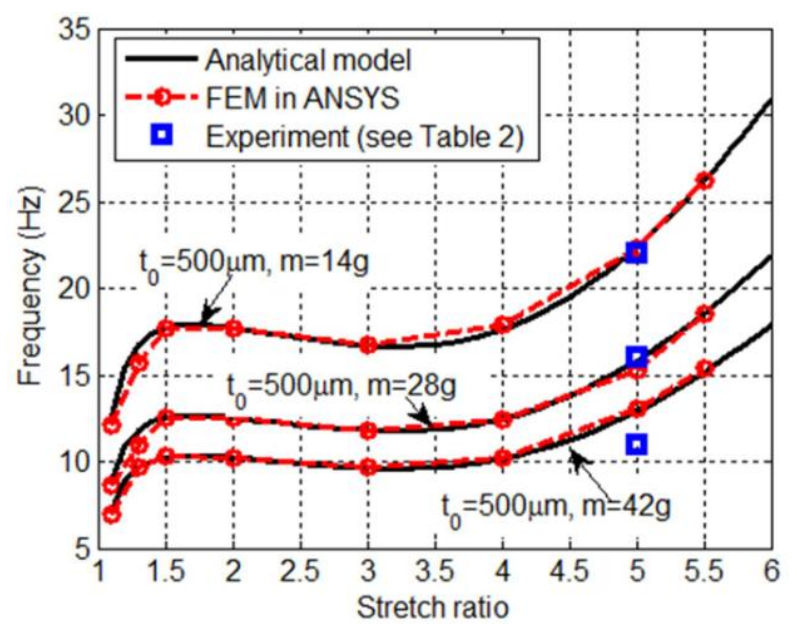

(a)

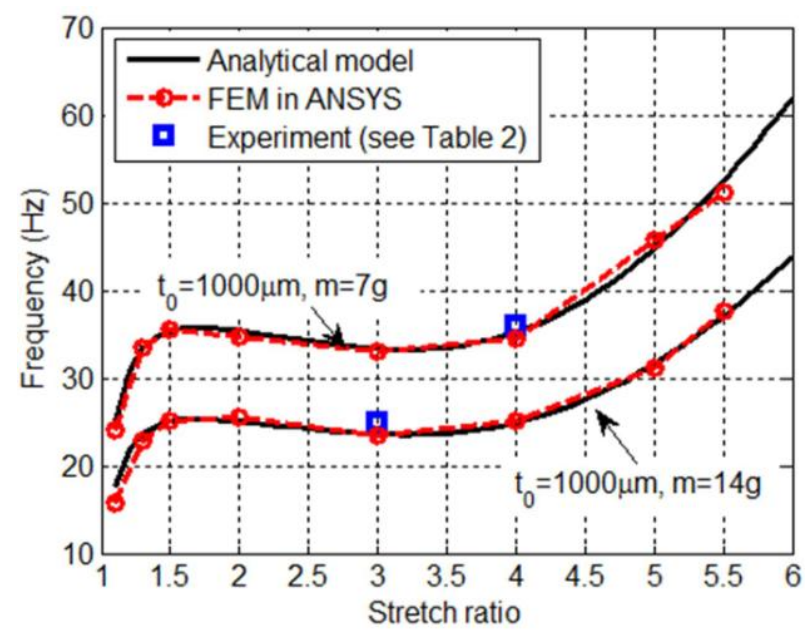

(b)

Fig 9. Comparison of analytical predictions (black lines), experimental data (squares) and FEM results (dashed lines) for the effective first mode natural frequency of a circular mass-loaded hyperelastic membrane as a function of stretch ratio subjected to mechanical stretch: (a) the initial (unstretched) membrane thickness is $500 \mu \mathrm{m}$; (b) the initial (unstretched) membrane thickness is $1000 \mu \mathrm{m}$. 


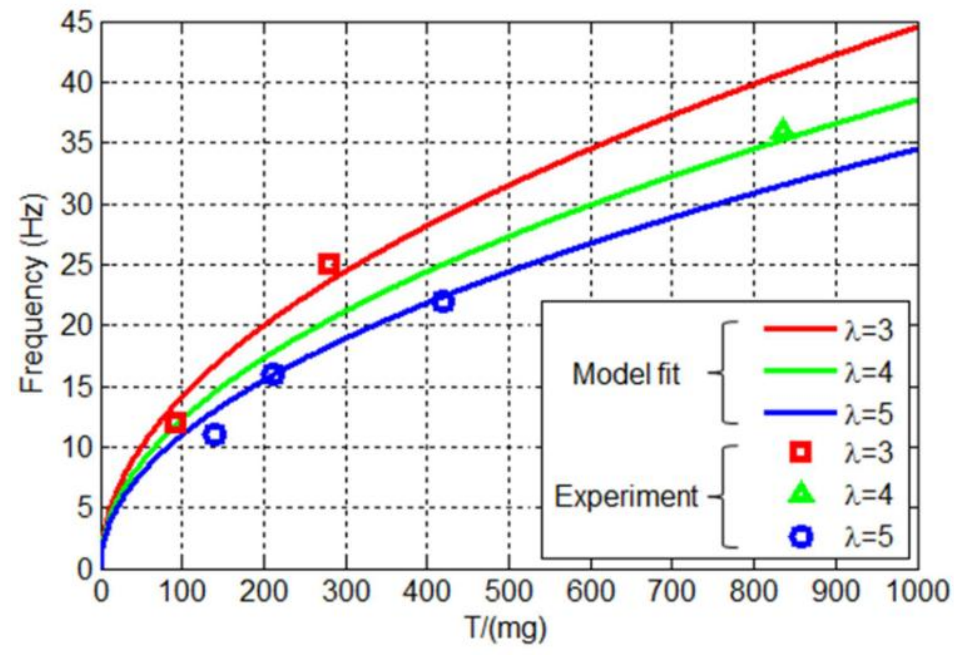

Fig 10. Comparison of analytical predictions and experimental data for the effective first mode natural frequency of a circular mass-loaded membrane subject to different stretch ratios. 
Table 1. Material parameters of hyperelastic membrane.

\begin{tabular}{cccc}
\hline \multicolumn{4}{c}{ Membrane (3M VHB 4910) } \\
\hline Symbol & Description & Value & Units \\
\hline$r_{0}$ & Initial radius & 19.05 & $\mathrm{~mm}$ \\
$t_{0}$ & Initial thickness & 1000 & $\mu \mathrm{m}$ \\
$\lambda$ & Stretch ratio & $1.1-5$ & unitless \\
$\varepsilon_{r}$ & Dielectric constant & 4.7 & unitless \\
$E$ & Young's modulus & 1.44 & $\mathrm{MPa}$ \\
$\alpha$ & Thermal expansion coefficient & $1.8 \times 10^{-4}$ & $\mathrm{~m} / \mathrm{m}-{ }^{\circ} \mathrm{C}$ \\
\hline$C_{10}$ & & 0.0693 & $\mathrm{MPa}$ \\
$C_{20}$ & Yeoh model & $-8.8 \times 10^{-4}$ & $\mathrm{MPa}$ \\
$C_{30}$ & & $16.7 \times 10^{-6}$ & $\mathrm{MPa}$ \\
\hline
\end{tabular}


Table 2. Experimental conditions for measurement of the tuned frequency of stretched hyperelastic $3 \mathrm{M}$ VHB 4910 membranes. The initial unstretched radius of all membranes is $19.05 \mathrm{~mm}$.

\begin{tabular}{ccccc}
\hline $\begin{array}{c}\text { Initial } \\
\text { thickness } \\
(\mu \mathrm{m})\end{array}$ & $\begin{array}{c}\text { Stretch } \\
\text { ratio }\end{array}$ & $\begin{array}{c}\text { Stretched } \\
\text { thickness } \\
(\mu \mathrm{m})\end{array}$ & $\begin{array}{c}\text { Added } \\
\text { mass }(\mathrm{g})\end{array}$ & $\begin{array}{c}\text { Tuned } \\
\text { frequency } \\
(\mathrm{Hz})\end{array}$ \\
\hline 500 & 5 & 20 & 14 & 22 \\
500 & 5 & 20 & 28 & 16 \\
500 & 5 & 20 & 42 & 11 \\
\hline 1000 & 4 & 60 & 7 & 36 \\
1000 & 3 & 111 & 14 & 25 \\
1000 & 3 & 111 & 42 & 12 \\
\hline
\end{tabular}

